\title{
Optimizing Patient Specific Medication Cassette Accuracy
}

\author{
Maria Paiva ${ }^{1 *}$ and Cathal Murphy ${ }^{2}$ \\ ${ }^{1}$ BSP, PharmD, BCPS Manager - Pediatric Medicine, Department of Clinical Operations, Sidra Medicine, Doha, Qatar, Adjunct Clinical Lecturer, College \\ of Pharmacy Qatar University Doha, Qatar \\ ${ }^{2}$ RN, BSc, Post-Grad Children's Nursing, Clinical Nurse Leader (CNL), Pediatric and Cardiac Intensive Care Unit Sidra Medicine, Doha, Qatar \\ ${ }^{*}$ Corresponding author: Maria Paiva, BSP, PharmD, BCPS Manager - Pediatric Medicine, Department of Clinical Operations, Sidra Medicine, Doha, Qatar, \\ Adjunct Clinical Lecturer, College of Pharmacy Qatar University Doha, Qatar
}

Received: June 18, 2020; Accepted: June 24, 2020; Published: July 08, 2020

\begin{abstract}
Due to fluctuation in clinical status, multiple medication changes may be implemented throughout a pediatric intensive care admission. An audit revealed multiple discrepancies between the active medication administration record (MAR) and patient specific medications stored on the unit. These discrepancies could have potentially led to medication administration errors. An operational definition of 'accuracy of compliance' and target was established by a working group. Utilizing "Plan Do Study Act" methodology, initiatives were implemented until the target of 95\% accuracy was achieved \& sustained. The most common reasons for inaccuracy were expired medications, discontinued medications, and incorrect patients' medications in the cassette. The most effective intervention was implementing medication cassette reconciliation with the MAR at shift change between primary clinical nurses.
\end{abstract}

Keywords: Patient specific medications, Cassette, Process improvement

\section{Introduction}

Sidra Medicine is a tertiary care academic hospital with a 24bed Pediatric Cardiac Intensive Care Unit (PCICU). This criticallyill cohort is diverse in age (neonates with cardiac morbidity up to 18 years of age) and complexity, resulting in the need for multiple patient specific medications to be prepared and stored in patient specific cassettes. Due to fluctuation in clinical status, multiple medication changes may be implemented throughout an ICU admission.

An audit in August 2019 revealed multiple discrepancies between the active medication administration record (MAR) and patient specific medications. These discrepancies could have potentially led to medication administration errors.

\section{Objectives}

The primary objective of this quality improvement initiative was to improve the accuracy of patient specific medication cassettes in the PCICU from $87 \%$ to $95 \%$. The secondary objective was to investigate if any medication errors reported in the event reporting system (Datix $\left.{ }^{\infty}\right)$ were linked to inaccurate contents in the medication cassettes.

\section{Methods}

Stakeholders from medical, nursing, allied health, and clinical operations were engaged; causes \& potential solutions discussed, and an action plan was developed. An operational definition of 'accuracy of compliance' established as each patient to have their specific medication cassette contain the exact list of medications on the active MAR. A target of $95 \%$ was chosen based on working group consensus and alignment with other hospital medication-related metrics. Utilizing "Plan Do Study Act" (PDSA) methodology, initiatives were implemented until the target of $95 \%$ accuracy was achieved \& sustained.

Initiatives included:

- September 2019: Clinical nurse leaders (CNLs) provided informal education to clinical nurses on the audit results and the importance of regularly checking the medication cassettes.

- October 2019: CNLs reminded clinical nurses at morning huddle prior to shift handover to review their medication cassettes against the MAR.

- November 2019: Implemented medication cassette reconciliation with the MAR at shift change between the outgoing and incoming primary clinical nurses.

- The event reporting system, Datix ${ }^{\oplus}$, was retroactively reviewed monthly after initiatives were implemented to identify any medication-related errors.

\section{Results}

Process improvement initiatives implemented increased the accuracy of the patient specific medication cassettes from an average of $87 \%$ to $99 \%$ by the end of November 2019 . Compliance above $95 \%$ has been sustained from November to date. When Datix ${ }^{\oplus}$ records were reviewed retroactively, none related to inaccurate medication cassette contents were reported. A run-chart with corresponding PDSA cycles is outlined in Figure 1. 


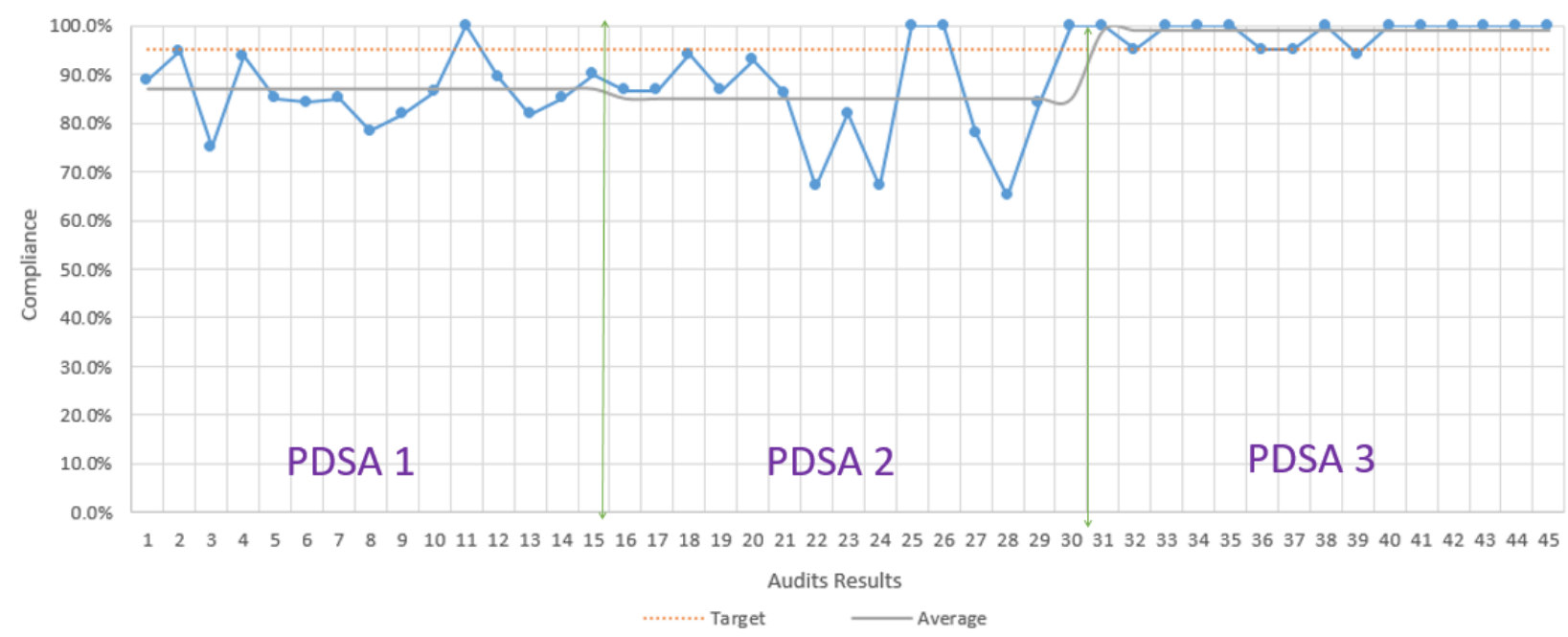

Figure 1: Outline of run-chart with corresponding PDSA cycles.

\section{Discussion and Conclusion}

The accuracy of medications in each patient specific cassette improved from an average of $87 \%$ to $99 \%$ after three PDSA cycles. The most common reasons for inaccuracy in descending order were expired medications, discontinued medications, and incorrect patients' medications in the cassette. Education and reinforcement of checking the cassettes at daily morning huddle had a modest impact on improving the metric. The most effective intervention was implementing medication cassette reconciliation with the MAR at shift change between primary clinical nurses. The impact of the interventions on medication errors reported in Datix ${ }^{\oplus}$ could not be assessed due to low reporting rate.

\section{Competing Interests}

The authors have no competing interests to declare.

\section{Funding}

No funding was received for this initiative or manuscript.

\section{Citation:}

